\title{
Validity of Diagnostic Codes for Identification of Psoriasis Patients in Korea
}

\author{
Seung Pil Ham, Jae Hong Oh, Hee Jae Park, Jong Uk Kim ${ }^{1}$ Ho Young Kim² , So Young Jung ${ }^{3}$, \\ Sun Young Choi ${ }^{4}$, Jung Eun Seol ${ }^{1}$, Hyojin Kim ${ }^{1}$, Myoung Shin $\mathrm{Kim}^{2}$, Un Ha Lee ${ }^{2}$, Mira Choi, \\ Hai-Jin Park
}

Department of Dermatology, Ilsan Paik Hospital, Inje University College of Medicine, Goyang, ${ }^{1}$ Department of Dermatology, Busan Paik Hospital, Inje University College of Medicine, Busan, ${ }^{2}$ Department of Dermatology, Sanggye Paik Hospital, Inje University College of Medicine, Seoul, ${ }^{3}$ Department of Dermatology, Haeundae Paik Hospital, Inje University College of Medicine, Busan, ${ }^{4}$ Department of Dermatology, Seoul Paik Hospital, Inje University College of Medicine, Seoul, Korea

Background: Recently, the number of nationwide medical researches on psoriasis using the National Health Insurance Service database has been on the rise. However, identification of psoriasis using diagnostic codes alone can lead to misclassification. Accuracy of the diagnostic codes and their concordance with medical records should be validated first to identify psoriasis patients correctly. Objective: To validate the diagnostic codes of psoriasis (International Classification of Diseases, 10th Revision L40) and to find the algorithm for the identification of psoriasis. Methods: We collected medical records of patients who received their first diagnostic codes of psoriasis during 5 years from five hospitals. Fifteen percent of psoriasis patients were randomly selected from each hospital. We performed a validation by reviewing medical records and compared 5 algorithms to identify the best algorithm. Results: Total of 538 cases were reviewed and classified as psoriasis $(n=368)$, not psoriasis $(n=159)$, and questionable $(n=11)$. The most accurate algorithm was including patients with $\geq 1$ visits with psoriasis as primary diagnostic

Received July 25, 2019, Revised November 21, 2019, Accepted for publication November 27, 2019

Corresponding author: Hai-Jin Park, Department of Dermatology, Inje University Ilsan Paik Hospital, 170 Juhwa-ro, Ilsanseo-gu, Goyang 10380, Korea. Tel: 82-31-910-7224, Fax: 82-31-910-7227, E-mail: stratum@paik. ac.kr

ORCID: https://orcid.org/0000-0002-9274-9371

This is an Open Access article distributed under the terms of the Creative Commons Attribution Non-Commercial License (http://creativecommons. org/licenses/by-nc/4.0) which permits unrestricted non-commercial use, distribution, and reproduction in any medium, provided the original work is properly cited.

Copyright (c) The Korean Dermatological Association and The Korean Society for Investigative Dermatology codes and prescription of vitamin $\mathrm{D}$ derivatives. Its positive predictive value was $96.5 \%$ (95\% confidence interval [Cl], $93.9 \% \sim 98.1 \%$ ), which was significantly higher than those of the algorithm, including patients with $\geq 1$ visits with psoriasis as primary diagnostic codes or including $\geq 1$ visits with diagnostic codes of psoriasis (primary or additional) $(91.0 \%$ and $69.8 \%)$. Sensitivity was $90.8 \%(95 \% \mathrm{Cl}, 87.2 \%$ $93.4 \%)$ and specificity was $92.5 \%(95 \% \mathrm{Cl}, 86.9 \%$ 95.9\%). Conclusion: Our study demonstrates a validated algorithm to identify psoriasis, which will be useful for the nationwide population-based study of psoriasis in Korea. (Ann Dermatol 32(2) $115 \sim 121,2020$ )

\section{-Keywords-}

Electronic medical record, International Classification of Disease Codes, National Health Insurance, Psoriasis

\section{INTRODUCTION}

Psoriasis is a chronic immune-mediated inflammatory disorder, which affects approximately $0.5 \% \sim 3 \%$ of the general population ${ }^{1}$. Recently, we saw a significant increase in the number of psoriasis researches using the nationwide health insurance and claims databases ${ }^{2}$. Population-based studies can facilitate research on the psoriasis-associated comorbidities, and their psychosocial and economic impact on the patients and produce more useful and qualified results ${ }^{1,3}$.

In Korea, two nationwide databases gathered respectively by the National Health Insurance System (NHIS) and the Health Insurance Review and Assessment Service (HIRA) 
cover nearly $98 \%$ of the Korean population. Researches based on insurance claims data identify psoriasis patients usually by using the diagnostic codes ${ }^{4,5}$. However, since electronic medical charts are not directly linked with the NHIS and HIRA databases, identifying patients with diagnostic codes alone can result in the misclassification of the concerned patients. According to a report from HIRA, the inconsistency rate between diagnostic codes used for insurance claims and the final diagnosis stated in the electronic medical records (EMR) varies from $23.6 \%$ to $61.4 \%$, depending on the diseases ${ }^{6}$. Therefore, in order to ensure the credibility of any research efforts using these databases, the accuracy of the diagnostic codes and their concordance with the EMR should be validated first.

In countries such as Taiwan ${ }^{7}$, Sweden $^{8}$, and Denmark ${ }^{9}$, which have an established nationwide or provincial healthcare database system, diagnostic code validation tests precede any population-based cohort studies of psoriasis. However, not much effort has been made to verify the validity of psoriasis codes used in cohort studies in Korea.

In this study, we aimed to validate psoriasis' diagnostic code used in insurance claims by comparing them with the final diagnosis in EMR. We also analyzed the validity of several algorithms using diagnostic codes and prescription data to develop the most accurate algorithm for the identification of psoriasis patients in Korea.

\section{MATERIALS AND METHODS}

\section{Data source}

This research is a retrospective, multicenter study participated by Inje University of Korea's five affiliated hospitals (four secondary- and one tertiary-level) located in three different cities of Seoul, Goyang, and Busan. It used the diagnostic codes from the International Classification of Diseases, 10th Revision (ICD-10) because both NHIS and HIRA databases are based on these codes. Psoriasis patient data, including their diagnostic codes, age, sex, and prescribed treatments (topical vitamin $D$ derivatives and narrow band ultraviolet B [UVB] phototherapy) was retrieved from each hospital's EMR. Topical vitamin D derivatives includes both single and combination agents with topical steroid.

This study was approved by the institutional review board of Inje University Hospitals: Ilsan Paik hospital (IRB no. 2018-02-010), Busan Paik hospital (IRB no. 2018-0125), Sanggye Paik hospital (IRB no. 2018-07-024), Haeundae Paik hospital (IRB no. 2018-07-005), and Seoul Paik hospital (IRB no. 2018-06-009).

\section{Study population}

We collected the medical records of outpatients who visited the dermatology department and were assigned a psoriasis code for the first time during 5 years from January 1, 2012 to December 31, 2016. The diagnostic codes which we considered as describing psoriasis were L40.0 (psoriasis vulgaris), L40.1 (generalized pustular psoriasis), L40.2 (acrodermatitis continua), L40.4 (guttate psoriasis), L40.5 (arthropathic psoriasis), L40.8 (other psoriasis) and L40.9 (psoriasis, unspecified) of ICD-10. The diagnostic codes of M07.0 (distal interphalangeal psoriatic arthropathy), M07.1 (arthritis mutilans), M07.2 (psoriatic spondylitis), M07.3 (psoriatic arthropathies) and M09.0 (juvenile arthritis in psoriasis) were also regarded as representing the same disease as the one by L40.5. Patients coded as L40.3 (palmoplantar psoriasis) were excluded as it can be easily confused with other diseases like palmoplantar keratoderma, pompholyx and hand eczema ${ }^{8}$. We also excluded the inpatient data as well as those from the other departments, including rheumatology.

\section{Psoriasis cases ascertainment and validation}

From each hospital, we randomly sampled $15 \%$ of the patients as described in the section above. Then, dermatology specialists of each hospital reviewed the EMR of the sampled patients. After reviewing all the sampled patients' medical records, clinical photos, pathologic reports, and prescribed drugs, we classified them into three groups: psoriasis, not psoriasis, and questionable to diagnose (Fig. 1). We compared positive predictive value (PPV), sensitivity, and specificity of several algorithms to identify psoriasis patients by using diagnostic codes and insurance claim data. We analyzed the type of psoriasis diagnostic code, whether psoriasis code was a primary diagnosis or additional diagnosis in each visit. Furthermore, we counted the number of visits in which the psoriasis was recorded as a primary diagnosis and additional diagnosis, respectively. ICD-10 guidelines divide patients' diagnosis into primary diagnosis and additional diagnosis. Primary diagnosis is defined as the condition primarily responsible for the treatment. Additional diagnosis is the conditions that develop or coexist with primary diagnosis and affects the management of the patients6. We also analyzed the algorithms including the vitamin $\mathrm{D}$ derivatives prescription data together with the diagnostic codes, because topical vitamin $\mathrm{D}$ derivatives are widely and prescribed specifically for psoriasis than for any other dermatosis. Total of five algorithms were analyzed: 1) $\geq 1$ visits with diagnostic codes of psoriasis (primary or additional); 2) $\geq 1$ visits with psoriasis as a primary diagnostic code; 3 ) $\geq 2$ 
visits with psoriasis as a primary diagnostic code; 4) $\geq 3$ visits with psoriasis as a primary diagnostic code; and 5) $\geq 1$ visits with psoriasis as a primary diagnostic code and prescription of vitamin D derivatives, including single and combination agents with topical steroids.

\section{Statistical analysis}

Collected data were analyzed by IBM SPSS Statistics ver. 22.0 (IBM corp., Armonk, NY, USA). A $p<0.05$ was regarded as statistically significant. PPV, sensitivity, and specificity were estimated with $95 \%$ confidence intervals $(\mathrm{Cl})$. As PPV is the percentage of patients captured by an algorithm who actually have the disease of interest, the algorithm with higher PPV can more successfully identify whether the patients do or do not have the target disease $^{10,11}$. Additionally, both sensitivity and specificity are concerned as secondary factors to determine a more valuable algorithm ${ }^{12}$.

\section{RESULTS}

\section{Characteristics of sampled patients}

The sampled data had a total of 3,587 patients who have either a primary or additional psoriasis diagnostic codes. Randomly selected 538 cases or $15.0 \%$ of the whole sample were reviewed. Table 1 shows the characteristics of those sampled patients. The data were comprised of 249 males (46.3\%) and 289 females (53.7\%). After reviewing the medical record of each patient, we classified 368 pa-

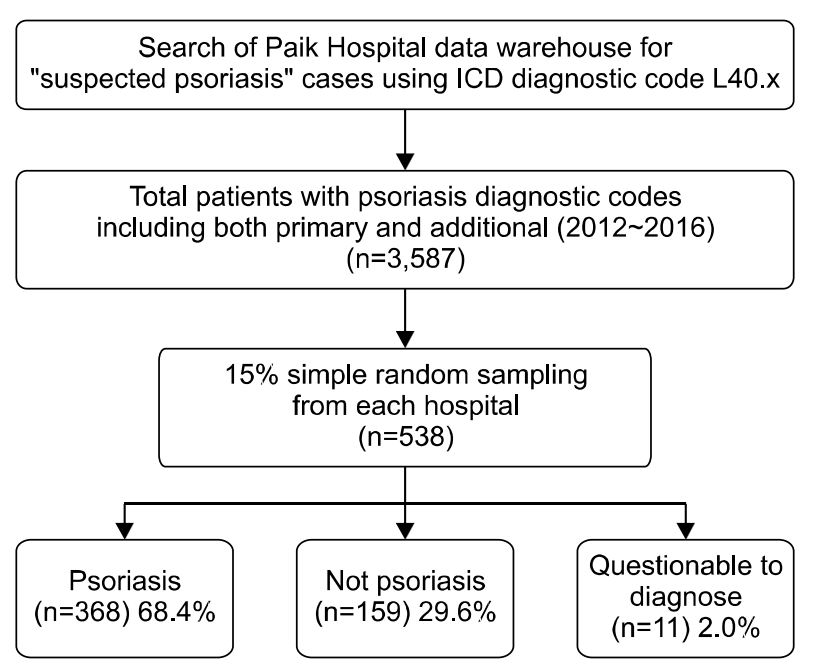

Fig. 1. Flow chart of study population selection. Total 3,587 patients with psoriasis diagnostic codes were included. Random samples of 538 cases were collected (15\% of total cases from each hospital). After the review, $368(68.4 \%)$ were psoriasis, 159 $(29.6 \%)$ were not psoriasis, and $11(2.0 \%)$ were questionable to diagnosis. ICD: International Classification of Diseases. tients $(68.4 \%)$ as psoriasis; $159(29.6 \%)$ as not psoriasis; and $11(2.0 \%)$ as questionable to diagnose. The most commonly used diagnostic code was L40.9 'psoriasis, unspecified' as found in the case of 284 patients (52.8\%), followed by L40.0 'psoriasis vulgaris' as in 188 patients $(34.9 \%)$, and L40.8 'other psoriasis' as in 36 patients (6.7\%). A total of 394 patients $(73.2 \%)$ had psoriasis as a primary diagnostic code, while 144 patients (26.8\%) had as additional diagnostic codes. While topical vitamin D derivatives, including single and combination agents, were prescribed to 448 patients (83.3\%), only 134 (24.9\%) were treated with narrowband UVB phototherapy.

\section{Analysis of algorithms}

We assessed the PPV, sensitivity, and specificity of each algorithm and compared one another. Since only 11 patients $(2.0 \%)$ were classified as questionable to diagnose after the medical chart review, they were excluded from

Table 1. Patient demographics and clinical characteristics $(n=538)$

\begin{tabular}{lrl}
\hline \multicolumn{1}{c}{ Variable } & Value \\
\hline Mean age (yr) & $45.4 \pm 17.3$ \\
Sex & $249(46.3)$ \\
Male & $289(53.7)$ \\
Female & \\
Used ICD-10 code & $188(34.9)$ \\
L40.0 Psoriasis vulgaris & $3(0.6)$ \\
L40.1 Generalized pustular psoriasis & $0(0.0)$ \\
L40.2 Acrodermatitis continua & $24(4.4)$ \\
L40.4 Guttate psoriasis & $3(0.6)$ \\
L40.5/M code arthropathic psoriasis & $36(6.7)$ \\
L40.8 Other psoriasis & $284(52.8)$ \\
L40.9 Psoriasis, unspecified & \\
Type of diagnostic code & $394(73.2)$ \\
Primary diagnosis & $144(26.8)$ \\
Additional diagnosis & \\
Diagnosis by review of medical record & $368(68.4)$ \\
Psoriasis & $159(29.6)$ \\
Not psoriasis & $11(2.0)$ \\
Questionable to diagnose & \\
Treatment with topical vitamin D derivatives & \\
Yes & $448(83.3)$ \\
No & $90(16.7)$ \\
Treatment with narrowband UVB phototherapy & \\
Yes & $134(24.9)$ \\
No & $404(75.1)$ \\
\hline
\end{tabular}

Values are presented as mean \pm standard deviation or number (\%). ICD-10: International Classification of Diseases, 10th Revision, UVB: ultraviolet B. M code: M07.0 Distal interphalangeal psoriatic arthropathy, M07.1 Arthritis mutilans, M07.2 Psoriatic spondylitis, M07.3 Psoriatic arthropathies, M09.0 Juvenile arthritis in psoriasis. ${ }^{\dagger}$ Topical vitamin $\mathrm{D}$ derivatives: include single and combination agents with topical steroids. 
Table 2. Comparing algorithm of primary diagnostic code with algorithm with both primary and additional diagnostic code

\begin{tabular}{cccc}
\hline Algorithm & $\begin{array}{c}\text { PPV \% } \\
(95 \% \mathrm{Cl})\end{array}$ & $\begin{array}{c}\text { Sensitivity \% } \\
(95 \% \mathrm{Cl})\end{array}$ & $\begin{array}{c}\text { Specificity \% } \\
(95 \% \mathrm{Cl})\end{array}$ \\
\hline$\geq 1$ visits with diagnostic codes of psoriasis (primary or additional) & 69.8 & 100 & - \\
$\geq 1$ visits with psoriasis as a primary diagnostic code & 91.0 & 96.2 & 78.0 \\
& $(87.6 \sim 93.6)$ & $(93.6 \sim 97.8)$ & $(70.6 \sim 84.0)$ \\
\hline
\end{tabular}

Values are presented as percentage. PPV: positive predictive value, Cl: confidential interval, -: not available.

Table 3. Validation of algorithms according to the number of visits with psoriasis as a primary diagnostic code

\begin{tabular}{|c|c|c|c|}
\hline Algorithm & $\begin{array}{c}\text { PPV \% } \\
(95 \% \quad \mathrm{Cl})\end{array}$ & $\begin{array}{c}\text { Sensitivity \% } \\
(95 \% \text { Cl) }\end{array}$ & $\begin{array}{c}\text { Specificity \% } \\
(95 \% \mathrm{Cl})\end{array}$ \\
\hline$\geq 1$ visits with psoriasis as a primary diagnostic code & $\begin{array}{c}91.0 \\
(87.6 \sim 93.6)\end{array}$ & $\begin{array}{c}96.2 \\
(93.6 \sim 97.8)\end{array}$ & $\begin{array}{c}78.0 \\
(70.6 \sim 84.0)\end{array}$ \\
\hline$\geq 2$ visits with psoriasis as a primary diagnostic code & $\begin{array}{c}95.4 \\
(92.3 \sim 97.4)\end{array}$ & $\begin{array}{c}79.6 \\
(75.1 \sim 83.5)\end{array}$ & $\begin{array}{c}91.2 \\
(85.4 \sim 94.9)\end{array}$ \\
\hline$\geq 3$ visits with psoriasis as a primary diagnostic code & $\begin{array}{c}96.9 \\
(93.3 \sim 98.6)\end{array}$ & $\begin{array}{c}58.4 \\
(53.2 \sim 63.5)\end{array}$ & $\begin{array}{c}95.6 \\
(90.8 \sim 98.1)\end{array}$ \\
\hline
\end{tabular}

Values are presented as percentage. PPV: positive predictive value, $\mathrm{Cl}$ : confidential interval.

Table 4. Validation and comparison of algorithm including prescription data of topical vitamin D derivatives

\begin{tabular}{lccc}
\multicolumn{1}{c}{ Algorithm } & $\begin{array}{c}\text { PPV } \% \\
(95 \% \mathrm{Cl})\end{array}$ & $\begin{array}{c}\text { Sensitivity \% } \\
(95 \% \mathrm{Cl})\end{array}$ & $\begin{array}{c}\text { Specificity \% } \\
(95 \% \mathrm{Cl})\end{array}$ \\
\hline$\geq 1$ visits with psoriasis as a primary diagnostic code & 91.0 & 96.2 & 78.0 \\
& $(87.6 \sim 93.6)$ & $(93.6 \sim 97.8)$ & $(70.6 \sim 84.0)$ \\
$\geq 1$ visits with psoriasis as a primary diagnostic code and & 96.5 & 90.8 & 92.5 \\
prescription of vitamin D & $(93.9 \sim 98.1)$ & $(87.2 \sim 93.4)$ & $(86.9 \sim 95.9)$ \\
\hline
\end{tabular}

Values are presented as percentage. PPV: positive predictive value, $\mathrm{Cl}$ : confidential interval, vitamin D: topical vitamin D derivatives including single and combination agents with steroids.

this study. The PPV of the algorithm including the patients with $\geq 1$ visits with psoriasis as a primary diagnostic code was much higher than that of the algorithm, including $\geq 1$ visits with diagnostic codes of psoriasis (primary or additional; $91.0 \%$ vs. $69.8 \%$ ) (Table 2 ).

The validation results of algorithms by the number of visits with psoriasis as a primary diagnostic code are shown in Table 3. Requiring $\geq 1$ visits with psoriasis as a primary diagnostic code revealed a PPV of $91.0 \%(95 \% \mathrm{Cl}$, 87.6 93.6) with sensitivity $96.2 \%(95 \% \mathrm{Cl}, 93.6 \sim 97.8)$ and specificity $78.0 \%(95 \% \mathrm{Cl}, 70.6 \sim 84.0)$. While the PPVs and specificity of the algorithms increased in proportion to the number of visits at which psoriasis was a primary diagnostic code, the sensitivity decreased according to the number of visits. The PPV of the algorithm, including patients with $\geq 3$ visits with psoriasis as a primary diagnostic code, was $96.9 \%(95 \% \mathrm{Cl}, 93.3 \sim 98.6)$ and the specificity was $95.6 \%(95 \% \mathrm{Cl}, 90.8 \sim 98.1)$, but the sensitivity was $58.4 \%(95 \% \mathrm{Cl}, 53.2 \sim 63.5)$.
We also analyzed the algorithms, including the prescription of topical vitamin D derivatives as single and combination agents with a topical steroid (Table 4). Algorithm including patients with $\geq 1$ visits with psoriasis as a primary diagnostic code and prescription of vitamin D derivatives showed higher PPV $(96.5 \%, 95 \% \mathrm{Cl}, 93.9 \sim$ 98.1) with sensitivity $90.8 \%(95 \% \mathrm{Cl}, 87.2 \sim 93.4)$ and specificity $92.5 \%(95 \% \mathrm{Cl}, 86.9 \sim 95.9)$ than the one including the patients with $\geq 1$ visits with psoriasis as a primary diagnostic code only $(91.0 \%, 95 \% \mathrm{Cl}, 87.6 \sim 93.6)$.

\section{DISCUSSION}

Nationwide health insurance databases provide population-based information of patients and healthcare services accumulated over a long period of time ${ }^{13}$. In South Korea, two nationwide databases gathered respectively by NHIS and HIRA provide population-based health insurance data, which cover almost $98 \%$ of the Korean pop- 
ulation $^{14-16}$. However, these databases have some limitations. First, as they were not established for medical researches, their structures were not explicitly designed to facilitate the identification of prevalence and incidence of a disease. Second, NHIS and HIRA databases have both primary and additional diagnoses along with the prescriptions and claims data, which makes it further challenging to identify a particular disease ${ }^{16}$.

Psoriasis researches using health insurance records are increasing around the world. The validation of diagnostic code is very important in the study using this data to reduce the risk of bias. Several countries, including the USA, Sweden, and the UK, performed studies to validate the algorithms to identify psoriasis patients by using their nationwide databases ${ }^{8,17-20}$. In the UK, Huerta et al. ${ }^{17}$ reported that the PPV was $82 \%$, when they selected patients who were referred to a specialist or hospitalized; received psoriasis treatment including topical and/or systemic; or was diagnosed with psoriasis one or more times during their research period. In Sweden, Löfvendahl et al. ${ }^{8}$ reported that when based on the data from the Skane Healthcare Register, requiring patients with $\geq 2$ diagnostic codes in specialized care could increase the PPV up to $100 \%$. Seminara et al. ${ }^{18}$ performed a validation study using Read Codes of the UK's Health Improvement Network. They validated an algorithm of $\geq 2$ Read Codes of psoriasis in other dates with high PPV (95\%) but lower sensitivity (74\%) and specificity $(67 \%)$.

Several nationwide psoriasis studies used the database of NHIS in Korea ${ }^{21}$. In these studies, researchers identified psoriasis patients by including those who had at least one diagnostic code of psoriasis without validation. However, identifying psoriasis patients in this way can cause bias because many non-psoriasis patients can be included in the study population ${ }^{4,5,21}$.

In our study, the algorithm, including $\geq 1$ visits with psoriasis as a primary diagnostic code showed a higher PPV than the one including $\geq 1$ visits with diagnostic codes of psoriasis (primary or additional) $(91.0 \%$ vs. $69.8 \%$ ). Generally, additional diagnosis is considered when the conditions develop or coexist with primary diagnosis and all other disease than primary diagnosis. Therefore, the inclusion of the patients who have psoriasis as an additional diagnostic code can raise the possibility of misclassification. The PPV of an algorithm increased proportionally to the frequency of visits with diagnostic codes of psoriasis, as found similarly in the Swedish study ${ }^{8}$. However, the sensitivity decreased significantly according to the number of visits. Because some patients visited only 1 or 2 times with psoriasis diagnostic code during the study period and some patients' diagnostic code changed from psoriasis to other diseases during follow-up. It can cause a decrease in sensitivity but an increase of specificity.

However, identifying patients with a primary diagnostic code alone still has some potential for misclassification. In other words, even if a patient has received a primary diagnostic code for psoriasis at the initial visit or follow-ups, the patient can still be false-positive if the diagnosis rendered is not final. Icen et al. ${ }^{19}$ and Ahlehoff et al. ${ }^{22}$ suggested an algorithm of combining the topical vitamin D derivatives prescription data with the diagnostic codes to improve the validity of the data. Our result also showed that the PPV hit the highest $(96.5 \%)$ when the algorithm included patients of $\geq 1$ visits with psoriasis as a primary diagnostic code and prescription of topical vitamin D derivatives. However, as our study includes the patients diagnosed as psoriasis by dermatology specialists at secondary or tertiary hospitals only, the prescription rate of vitamin $\mathrm{D}$ derivatives can be higher than the cases in which the patients diagnosed by general physicians, rheumatologists, or dermatologists in primary medical care are included. Therefore, the algorithm, including patients with $\geq 1$ visits with psoriasis as a primary diagnostic code, could be an option for the studies, including general physicians and dermatologists in primary medical care, to increase the sensitivity of the algorithm. In our study, the sensitivity of the algorithm, including patients with $\geq 1$ visits with psoriasis as a primary diagnostic code was $96.2 \%$, which was higher than that of the algorithm included the prescription data of topical vitamin $\mathrm{D}$ derivatives $(90.8 \%)$.

Moreover, in our study, patients treated with phototherapy were fewer than we expected $(24.9 \%)$. Therefore, incorporating phototherapy into the algorithm may result in a significant loss of actual psoriasis patients, especially those with mild symptoms.

Meanwhile, this study should be read with the following limitations in mind. First, confirmation of psoriasis diagnosis was based on the review of medical record, which is subject to the uncertainty and bias of retrospective review. Second, this study used the data from the dermatology department of a general hospital only, which means that the diagnoses rendered by general physicians, rheumatologists, or dermatologists in primary medical care were not considered. Notwithstanding these limitations, however, this study is meaningful in many ways. First, it is the nation's first report on the validity of diagnostic codes used for the identification of psoriasis patients in Korea. Second, as a multi-centered study using the data from different cities of the country, it produced a result neutral to the selection bias and geographical differences.

In conclusion, the algorithm, including patients with $\geq 1$ 
visits with psoriasis as a primary diagnostic code and prescription of vitamin D derivatives, is the best-validated algorithm to identify psoriasis patients with high PPV $(96.5 \%)$ and high specificity $(92.5 \%)$. Furthermore, the algorithm including patients with $\geq 1$ visits with primary diagnostic code of psoriasis, is another option when the study needs higher sensitivity or includes the diagnoses rendered by general physicians, rheumatologists, or dermatologists in primary medical care because it also has relatively high PPV (91.0\%) and sensitivity (96.2\%). These algorithms can help enhance the credibility of nationwide medical researches on psoriasis, which obtain their data from either NHIS or HIRA database.

\section{CONFLICTS OF INTEREST}

The authors have nothing to disclose.

\section{ORCID}

Seung Pil Ham, https://orcid.org/0000-0002-4472-2043

Jae Hong Oh, https://orcid.org/0000-0003-3394-860X

Hee Jae Park, https://orcid.org/0000-0002-3998-9042

Jong Uk Kim, https://orcid.org/0000-0001-8430-0323

Ho Young Kim, https://orcid.org/0000-0002-1029-3287

So Young Jung, https://orcid.org/0000-0003-0155-5473

Sun Young Choi, https://orcid.org/0000-0003-0248-7708

Jung Eun Seol, https://orcid.org/0000-0002-3029-9635

Hyojin Kim, https://orcid.org/0000-0003-0987-4938

Myoung Shin Kim, https://orcid.org/0000-0002-0660-8098

Un Ha Lee, https://orcid.org/0000-0003-1626-5583

Mira Choi, https://orcid.org/0000-0003-2464-9675

Hai-Jin Park, https://orcid.org/0000-0002-9274-9371

\section{REFERENCES}

1. Parisi R, Symmons DP, Griffiths CE, Ashcroft DM; Identification and Management of Psoriasis and Associated ComorbidiTy (IMPACT) Project Team. Global epidemiology of psoriasis: a systematic review of incidence and prevalence. J Invest Dermatol 2013;133:377-385.

2. Wilchesky M, Tamblyn RM, Huang A. Validation of diagnostic codes within medical services claims. J Clin Epidemiol 2004;57:131-141.

3. Kim ES, Han K, Kim MK, Park YM, Baek KH, Moon SD, et al. Impact of metabolic status on the incidence of psoriasis: a Korean nationwide cohort study. Sci Rep 2017;7:1989.

4. Han JH, Lee JH, Han KD, Seo HM, Bang CH, Park YM, et al. Epidemiology and medication trends in patients with psoriasis: a nationwide population-based cohort study from Korea. Acta Derm Venereol 2018;98:396-400.

5. Lee JY, Kang S, Park JS, Jo SJ. Prevalence of psoriasis in
Korea: a population-based epidemiological study using the Korean National Health Insurance Database. Ann Dermatol 2017;29:761-767.

6. Park BJ, Suh SW, Sung JH, Park GD, Kim SH. Improvement plan for validity of health insurance disease code and establishment of data application plan. Seoul: Health Insurance Review Agency Research Service, 2003.

7. Lee MS, Yeh YC, Chang YT, Lai MS. All-cause and cause-specific mortality in patients with psoriasis in Taiwan: a nationwide population-based study. J Invest Dermatol 2017;137:1468-1473.

8. Löfvendahl S, Theander E, Svensson $\AA$, Carlsson KS, Englund M, Petersson IF. Validity of diagnostic codes and prevalence of physician-diagnosed psoriasis and psoriatic arthritis in southern Sweden-a population-based register study. PLoS One 2014;9:e98024.

9. Ahlehoff $\mathrm{O}$, Gislason $\mathrm{GH}$, Jørgensen $\mathrm{CH}$, Lindhardsen J, Charlot $\mathrm{M}$, Olesen JB, et al. Psoriasis and risk of atrial fibrillation and ischaemic stroke: a Danish nationwide cohort study. Eur Heart J 2012;33:2054-2064.

10. Trevethan R. Sensitivity, specificity, and predictive values: foundations, pliabilities, and pitfalls in research and practice. Front Public Health 2017; 5:307.

11. Parikh R, Mathai A, Parikh S, Chandra Sekhar G, Thomas R. Understanding and using sensitivity, specificity and predictive values. Indian J Ophthalmol 2008;56:45-50.

12. Hawass NE. Comparing the sensitivities and specificities of two diagnostic procedures performed on the same group of patients. Br J Radiol 1997;70:360-366.

13. Raghupathi W, Raghupathi V. Big data analytics in healthcare: promise and potential. Health Inf Sci Syst 2014;2:3.

14. Kim JA, Yoon S, Kim LY, Kim DS. Towards actualizing the value potential of Korea Health Insurance Review and Assessment (HIRA) data as a resource for health research: strengths, limitations, applications, and strategies for optimal use of HIRA data. J Korean Med Sci 2017;32:718-728.

15. Kim L, Kim JA, Kim S. A guide for the utilization of Health Insurance Review and Assessment service national patient samples. Epidemiol Health 2014;36:e2014008.

16. Cheol Seong S, Kim YY, Khang YH, Heon Park J, Kang HJ, Lee $\mathrm{H}$, et al. Data resource profile: the national health information database of the National Health Insurance Service in South Korea. Int J Epidemiol 2017;46:799-800.

17. Huerta C, Rivero E, Rodríguez LA. Incidence and risk factors for psoriasis in the general population. Arch Dermatol 2007; 143:1559-1565.

18. Seminara NM, Abuabara K, Shin DB, Langan SM, Kimmel $\mathrm{SE}$, Margolis $\mathrm{D}$, et al. Validity of The Health Improvement Network (THIN) for the study of psoriasis. Br J Dermatol 2011;164:602-609.

19. Icen M, Crowson CS, McEvoy MT, Gabriel SE, Maradit Kremers $\mathrm{H}$. Potential misclassification of patients with psoriasis in electronic databases. J Am Acad Dermatol 2008;59: 981-985.

20. Asgari MM, Wu JJ, Gelfand JM, Salman C, Curtis JR, Harrold LR, et al. Validity of diagnostic codes and prevalence of psoriasis and psoriatic arthritis in a managed care pop- 
ulation, 1996-2009. Pharmacoepidemiol Drug Saf 2013;22: 842-849.

21. Oh EH, Ro YS, Kim JE. Epidemiology and cardiovascular comorbidities in patients with psoriasis: a Korean nationwide population-based cohort study. J Dermatol 2017;44:
621-629.

22. Ahlehoff $\mathrm{O}$, Gislason $\mathrm{GH}$, Charlot $\mathrm{M}$, Jørgensen $\mathrm{CH}$, Lindhardsen J, Olesen JB, et al. Psoriasis is associated with clinically significant cardiovascular risk: a Danish nationwide cohort study. J Intern Med 2011;270:147-157. 